University of Nebraska - Lincoln

DigitalCommons@University of Nebraska - Lincoln

\title{
Regional Differences in Cervical Cancer Incidence Among American Indians and Alaska Natives, 1999-2004
}

Thomas M. Becker

Oregon Health \& Science University School of Medicine, beckert@ohsu.edu

David K. Espey

Centers for Disease Control and Prevention

Herschel W. Lawson

Centers for Disease Control and Prevention

Mona Saraiya

Centers for Disease Control and Prevention

Melissa A. Jim

Centers for Disease Control and Prevention

See next page for additional authors

Follow this and additional works at: https://digitalcommons.unl.edu/publichealthresources

Part of the Public Health Commons

Becker, Thomas M.; Espey, David K.; Lawson, Herschel W.; Saraiya, Mona; Jim, Melissa A.; and Waxman, Alan G., "Regional Differences in Cervical Cancer Incidence Among American Indians and Alaska Natives, 1999-2004" (2008). Public Health Resources. 234.

https://digitalcommons.unl.edu/publichealthresources/234

This Article is brought to you for free and open access by the Public Health Resources at DigitalCommons@University of Nebraska - Lincoln. It has been accepted for inclusion in Public Health Resources by an authorized administrator of DigitalCommons@University of Nebraska - Lincoln. 
Authors

Thomas M. Becker, David K. Espey, Herschel W. Lawson, Mona Saraiya, Melissa A. Jim, and Alan G. Waxman 


\section{An Update on Cancer in American Indians and Alaska Natives, 1999-2004}

Supplement to Cancer

\section{Regional Differences in Cervical Cancer Incidence Among American Indians and Alaska Natives, 1999-2004}

\author{
Thomas M. Becker, MD, PhD ${ }^{1}$ \\ David K. Espey, ${ }^{2}{ }^{2}$ \\ Herschel W. Lawson, $\mathrm{MD}^{2}$ \\ Mona Saraiya, $\mathrm{MD}, \mathrm{MPH}^{2}$ \\ Melissa A. Jim, $\mathrm{MPH}^{2}$ \\ Alan G. Waxman, MD, $\mathrm{MPH}^{3}$ \\ ${ }^{1}$ Department of Public Health and Preventive \\ Medicine, Oregon Health \& Science University \\ School of Medicine, Portland, Oregon. \\ ${ }^{2}$ Division of Cancer Prevention and Control, \\ National Center for Chronic Disease Prevention \\ and Health Promotion, Centers for Disease Con- \\ trol and Prevention, Atlanta, Georgia. \\ ${ }^{3}$ Department of Obstetrics and Gynecology, Uni- \\ versity of New Mexico Health Sciences Center Al- \\ buquerque, New Mexico.
}

This supplement was sponsored by Cooperative Agreement Number U50 DP424071-04 from the Centers for Disease Control and Prevention, Division of Cancer Prevention and Control.

The findings and conclusions in this report are those of the authors and do not necessarily represent the official position of the Centers for Disease Control and Prevention.

Address for reprints: Thomas Becker, MD, Department of Public Health and Preventive Medicine, Oregon Health \& Science University School of Medicine, Portland, OR 97202-3098; Fax: (503) 494-7536; E-mail: beckert@ohsu.edu

Received May 5, 2008; accepted June 3, 2008.

${ }^{*}$ This article is a US Government work and, as such, is in the public domain in the United States of America.
BACKGROUND. Reports from limited geographic regions indicate higher rates of cervical cancer incidence in American Indian and Alaska Native (AI/AN) women than in women of other races. However, accurate determinations of cervical cancer incidence in AI/AN women have been hampered by racial misclassification in central cancer registries.

METHODS. The authors linked data from cancer registries participating in the National Program of Cancer Registries (NPCR) and the Surveillance, Epidemiology, and End Results (SEER) Program with Indian Health Service (IHS) enrollment records to improve identification of AI/AN race. NPCR and SEER data were combined to estimate annualized age-adjusted rates (expressed per 100,000 persons) for the diagnosis years 1999 to 2004. Analyses focused on counties known to have less racial misclassification, and results were stratified by IHS Region. Approximately $56 \%$ of AI/ANs in the US reside in these counties. The authors examined overall and age-specific incidence rates and stage at diagnosis for AI/ AN women compared with non-Hispanic white (NHW) women.

RESULTS. Invasive cervical cancer incidence rates among AI/AN women varied nearly 2 -fold across IHS regions, with the highest rates reported in the Southern Plains (14.1) and Northern Plains (12.5); the lowest rates were in the Eastern region and the Pacific Coast. Overall, AI/AN women had higher rates of cervical cancer than NHW women and were more likely to be diagnosed with later stage disease.

CONCLUSIONS. The wide regional variation of invasive cervical cancer incidence indicates an important need for health services research regarding cervical cancer screening and prevention education as well as policy development regarding human papillomavirus vaccine use, particularly in the regions with high incidence rates. Cancer 2008;113(5 suppl):1234-43. Published 2008 by the American Cancer Society.*

KEYWORDS: American Indian/Alaska Native, cervical cancer, surveillance, incidence.

드 or all cancer sites combined, the majority of American Indian and Alaska Native (AI/AN) populations have exhibited rates lower than or similar to those of non-Hispanic white (NHW) populations or members of all racial groups combined. ${ }^{1-4}$ However, for cervical cancer and for preinvasive cervical lesions, prior investigations have found AI/AN women to have higher rates than other populations. ${ }^{1,5-9}$ Furthermore, the majority of data regarding cervical 
TABLE 1

Cervical Cancer Incidence by Indian Health Service Region for American Indians/Alaska Natives ${ }^{\mathrm{a}}$ and Non-Hispanic Whites, US, 1999 to 2004

\begin{tabular}{|c|c|c|c|c|c|c|c|c|c|c|c|c|}
\hline \multirow[b]{2}{*}{ IHS Region } & \multicolumn{6}{|c|}{ CHSDA Counties } & \multicolumn{6}{|c|}{ All Counties } \\
\hline & $\begin{array}{l}\text { AI/AN } \\
\text { Count }\end{array}$ & $\begin{array}{l}\text { AI/AN } \\
\text { Rate }^{\text {b }}\end{array}$ & $\begin{array}{l}95 \% \text { CI for } \\
\text { AI/AN Rate }\end{array}$ & $\begin{array}{l}\text { NHW } \\
\text { Rate }^{\text {b }}\end{array}$ & $\begin{array}{l}\text { Rate Ratioc } \\
\text { (AI/AN:NHW) }\end{array}$ & $\begin{array}{l}95 \% \text { CI for } \\
\text { Rate Ratio }\end{array}$ & $\begin{array}{l}\text { AI/AN } \\
\text { Count }\end{array}$ & $\begin{array}{l}\text { AI/AN } \\
\text { Rate }^{\text {b }}\end{array}$ & $\begin{array}{l}95 \% \text { CI for } \\
\text { AI/AN Rate }\end{array}$ & $\begin{array}{l}\text { NHW } \\
\text { Rate }^{b}\end{array}$ & $\begin{array}{l}\text { Rate Ratio } \\
\text { (AI/AN:NHW) }\end{array}$ & $\begin{array}{l}95 \% \text { CI for } \\
\text { Rate Ratio }\end{array}$ \\
\hline Northern Plains & 69 & 12.5 & $9.6-16.1$ & 7.4 & $1.69^{\mathrm{d}}$ & $1.29-2.18$ & 97 & 9.7 & $7.7-12.0$ & 7.6 & $1.28^{\mathrm{d}}$ & $1.02-1.58$ \\
\hline Alaska $^{\mathrm{e}}$ & 21 & 8.4 & $5.1-13.2$ & 6.2 & 1.37 & $0.77-2.29$ & 21 & 8.4 & $5.1-13.2$ & 6.2 & 1.37 & $0.77-2.29$ \\
\hline Southern Plains & 117 & 14.1 & $11.6-16.9$ & 9.1 & $1.54^{\mathrm{d}}$ & $1.25-1.87$ & 136 & 11.1 & $9.3-13.2$ & 8.6 & $1.30^{\mathrm{d}}$ & $1.08-1.54$ \\
\hline Pacific Coast & 65 & 6.9 & $5.2-8.9$ & 7.0 & 0.98 & $0.74-1.27$ & 85 & 4.9 & $3.9-6.1$ & 7.0 & $0.70^{\mathrm{d}}$ & $0.55-0.88$ \\
\hline East & 15 & 7.1 & $3.9-11.8$ & 7.3 & 0.97 & $0.53-1.62$ & 63 & 4.0 & $3.0-5.2$ & 8.0 & $0.50^{\mathrm{d}}$ & $0.38-0.65$ \\
\hline Southwest & 94 & 7.8 & $6.2-9.6$ & 7.3 & 1.07 & $0.85-1.32$ & 100 & 7.3 & $5.9-8.9$ & 6.8 & 1.08 & $0.86-1.32$ \\
\hline Total & 381 & 9.4 & $8.5-10.4$ & 7.4 & $1.28^{\mathrm{d}}$ & $1.15-1.42$ & 502 & 6.9 & $6.3-7.6$ & 7.7 & $0.90^{\mathrm{d}}$ & $0.82-0.99$ \\
\hline
\end{tabular}

Source: Cancer registries in the Centers for Disease Control and Prevention's National Program of Cancer Registries (NPCR) and/or the National Cancer Institute's Surveillance, Epidemiology and End Results (SEER) program.

CHSDA indicates Contract Health Service Delivery Areas; IHS, Indian Health Service; AI/AN, American Indians/Alaska Natives; 95\% CI, 95\% confidence interval; NHW, non-Hispanic whites.

${ }^{a}$ AI/AN race is reported by NPCR and SEER registries or through linkage with the IHS patient registration database. AI/AN persons of Hispanic origin are included.

${ }^{\mathrm{b}}$ Rates are per 100,000 persons and are age-adjusted to the 2000 US standard population (19 age groups).

${ }^{\mathrm{c}}$ Rate ratios are calculated in SEER ${ }^{\mathrm{a}}$ Stat prior to rounding of rates and may not equal rate ratios calculated from rates presented in the table.

${ }^{\mathrm{d}}$ Rate ratio is statistically significant $(P<.05)$.

e Rates and rate ratios for Alaska in the CHSDA counties section is the same as those in the All Counties section because all counties in Alaska are CHSDA counties.

Years of data and registries used: 1999 to 2004 (41 states and the District of Columbia; ${ }^{*}$ indicates states with at least 1 county designated as CHSDA): Alaska, ${ }^{*}$ Alabama, ${ }^{*}$ Arkansas, Arizona, ${ }^{*}$ California, ${ }^{*}$ Colorado, ${ }^{*}$ Connecticut, ${ }^{*}$ District of Columbia, Delaware, Florida, ${ }^{*}$ Georgia, Hawaii, Iowa, ${ }^{*}$ Idaho, ${ }^{*}$ Illinois, Indiana, ${ }^{*}$ Kentucky, , Louisiana, ${ }^{*}$ Massachusetts, ${ }^{*}$ Maine, ${ }^{*}$ Michigan, ${ }^{*}$ Minnesota, ${ }^{*}$ Missouri, Montana, ${ }^{*}$ North Carolina, ${ }^{*}$ Nebraska, ${ }^{*}$ New Hampshire, New Jersey, New Mexico, ${ }^{*}$ Nevada, ${ }^{*}$ New York, ${ }^{*}$ Ohio, Oklahoma, ${ }^{*}$ Oregon, ${ }^{*}$ Pennsylvania, ${ }^{*}$ Rhode Island, ${ }^{*}$ Texas, ${ }^{*}$ Utah, ${ }^{*}$ Washington, ${ }^{*}$ Wisconsin, ${ }^{*}$ West Virginia, and Wyoming*; 1999 and 2002 to 2004: North Dakota*; 2001 to 2004: South Dakota*; 2003 to 2004: Mississippi* and Virginia; 2004: Tennessee.

Percent regional coverage of AI/AN in CHSDA counties to AI/AN in all counties: Alaska: 100\%; East: 13.1\%; Northern Plains: 59.0\%; Southern Plains: 64.1\%; Pacific Coast: 55.6\%; Southwest: 87.5.1\%.

cancer mortality in AI/AN women published to date demonstrated substantially higher rates in comparison to NHW women..$^{5,8,10,11}$ This discrepancy has been most dramatically demonstrated among AI/AN women in the Southwest compared with other populations in the Southwest, through data collected by the New Mexico Tumor Registry, a long-standing Surveillance, Epidemiology, and End Results (SEER) site that operates with a minimum of racial misclassification. ${ }^{5,6,12}$ Although rates for invasive cervical cancer in AI/AN women in New Mexico and Alaska have been declining in recent years, ${ }^{13,14}$ rates for preinvasive lesions have remained high in some groups. ${ }^{5,9,15-17}$

Despite studies from limited geographic regions, AI/AN women have often been excluded from national and state reporting on cervical cancer because of race misclassification in central cancer registries. ${ }^{18-21}$ The purpose of the current study was to improve our characterization of invasive cervical cancer incidence and stage at diagnosis in AI/AN women relative to NHW women, using techniques to minimize the effect of race misclassification in cancer surveillance data.

\section{MATERIALS AND METHODS}

Detailed descriptions of the data sources and methods used for this analysis are found in another article in this supplement. ${ }^{22}$

\section{Cancer Cases}

US state and regional population-based cancer registries collect information on new cancer diagnoses. ${ }^{23}$ They participate in the National Program of Cancer Registries (NPCR) of the Centers for Disease Control and Prevention (CDC), the SEER Program of the National Cancer Institute (NCI), or both. ${ }^{24}$ Primary cancer site and histology data are coded according to the International Classification of Diseases for Oncology (ICD-O) edition in use at the time of diagnosis and converted to the third edition. ${ }^{25}$ For this study, cervical cancer incidence data generally refer to invasive cancers (ICD-O-3 C530-C539); lymphomas originating in the lymphatic tissue of the cervix, other histologies involving hematopoietic diseases, mesotheliomas, and Kaposi sarcomas were excluded (M9590-9989, 9050-9055, 9140). We did not examine in situ cervical tumors, which have not been reportable to NPCR and SEER since 1996. All cervical cancer data included in this study have been reviewed by cancer registrars, and meet the US Cancer Statistics standards ${ }^{24}$; the states that met quality criteria and that are included in the analysis are listed in the footnotes of Table 1.

Coding race for AI/AN cancer cases in this report combines information from 2 sources: 1) data linkages with the Indian Health Service (IHS) patient registration database, developed because the IHS 
provides medical services to AI/AN persons who are members of federally recognized tribes; and 2) the multiple race fields in central cancer registry records. ${ }^{26}$ To reduce the misclassification of AI/AN race, all case records from each state were linked with the IHS patient registration database to identify AI/AN cases misclassified as another race. Linkages were conducted using LinkPlus, a probabilistic linkage software program developed by the CDC that was applied to key patient identifiers (social security number, first name, last name, middle initial, date of birth, and date of death). ${ }^{27}$ Possible matches, requiring manual review, were examined independently by 2 reviewers, and when necessary, adjudicated by a third reviewer. The information from the linkages was then combined with the multiple race fields coded in cancer registry records. Further details regarding the linkage and coding rules for multiple races are described elsewhere in this supplement. ${ }^{22}$

Contract Health Service Delivery Area (CHSDA) counties and IHS regions were geographic factors used to characterize further the burden of cancer incidence in the AI/AN population. CHSDA counties, in general, contain federally recognized tribal lands or are adjacent to tribal lands. The proportions of AI/AN persons in relation to total population are higher in CHSDA counties than in non-CHSDA counties, and CHSDA counties demonstrate less race misclassification for AI/ANs. ${ }^{28}$ Data are presented for CHSDA counties and for all counties; however, because the information is believed to be more accurate for AI/AN persons living in CHSDA counties, the focus of the analyses is on AI/AN residing in CHSDA counties. The analysis of AI/AN data by IHS region (Alaska, Pacific Coast, Northern Plains, Southern Plains, Southwest, and East) conforms with known regional patterns of specific health outcomes and disease risk factors for AI/AN. ${ }^{11,29}$ A map depicting the IHS regions and indicating states and CHSDA counties included in the analysis is presented in Figure 1 . Approximately $56 \%$ of the US AI/AN population reside in CHSDA counties. This proportion varies by IHS region, from $15.4 \%$ reported in the East to $100 \%$ in Alaska. Additional details regarding CHSDA counties and IHS regions are provided elsewhere. ${ }^{22}$

Disease stage data for this report spanned changes in SEER summary stage coding. Stage was coded according to SEER summary stage 1977 rules for diagnosis years 1999 to 2000 and to SEER summary stage 2000 rules for 2001 to 2003; collaborative stage data, first reported for 2004, were not available for analysis. Because the 2 staging systems are comparable for cervical cancer, ${ }^{30,31}$ we combined 1999 to 2003 data in the stage analysis.

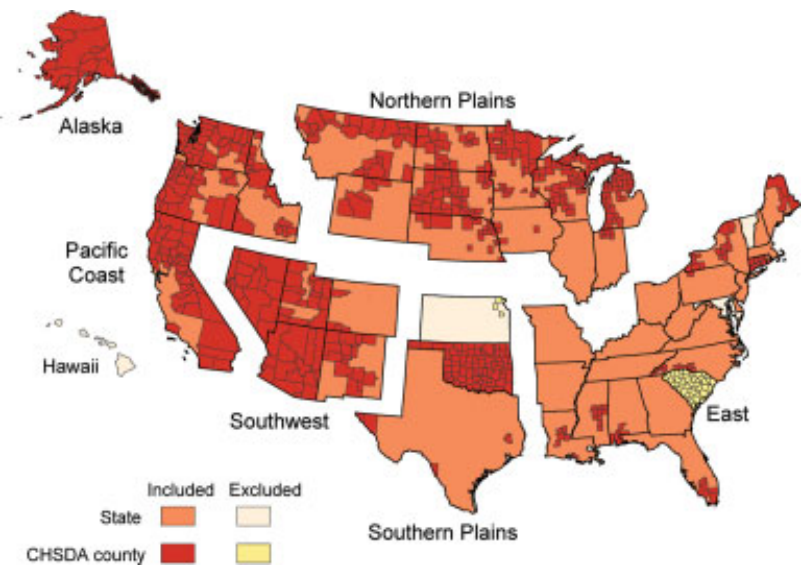

FIGURE 1. States and Contract Health Service Delivery Areas (CHSDA) counties by Indian Health Service region.

\section{Population Estimates}

Using population estimates from the Census Bureau and the CDC's National Center for Health Statistics, ${ }^{32}$ the NCI makes additional refinements regarding race and county geographic codes; these estimates were used as denominators in this report. ${ }^{22,33}$

\section{Statistical Analyses}

Two sets of statistics are provided for AI/AN and NHW women: 1) data from all counties in 46 states and the District of Columbia (referred to as 'All Counties'), and 2) data from counties designated as CHSDA. In addition, All Counties data and CHSDA counties data are provided for each IHS region. The results described in the text refer to persons who reside in CHSDA counties unless otherwise noted. Additional information concerning cases and population coverage is available elsewhere in this supplement. $^{22}$

For all AI/AN and NHW populations, cancer incidence rates were expressed per 100,000 persons and were age-adjusted by 19 age groups $(<1$ year, $1-4,5$ $9, \ldots, 80-84$, and $85+$ years) to the 2000 US standard population. Percent distributions for stage of disease at diagnosis are also age-adjusted. For all analyses, exact counts were suppressed when the category of interest contained $\leq 5$ cases. The age groups for the cervical cancer analysis (15-29, 30-49, 50-64, and $65+$ years) were selected with consideration of screening recommendations and other age-related influences on screening and prevention, such as Medicare benefits for those aged $\geq 65$ years.

Using the age-adjusted incidence rates, standardized rate ratios (RRs) were calculated for AI/AN women using NHW rates for comparison. Confidence 
TABLE 2

Invasive Cervical Cancer Incidence Rates ${ }^{\mathrm{a}}$ by Age and Indian Health Service Region for American Indians/Alaska Natives and Non-Hispanic Whites, CHSDA Counties, US, 1999 to 2004

\begin{tabular}{|c|c|c|c|c|c|c|c|c|c|c|c|c|c|c|c|c|}
\hline \multirow[b]{2}{*}{ IHS Region } & \multicolumn{4}{|c|}{ 15-29 Years } & \multicolumn{4}{|c|}{ 30-49 Years } & \multicolumn{4}{|c|}{ 50-64 Years } & \multicolumn{4}{|c|}{$65+$ Years } \\
\hline & Count & $\begin{array}{l}\text { Cases, } \\
\%^{\mathrm{a}}\end{array}$ & Rate $^{b}$ & $\begin{array}{l}95 \% \\
\text { CI }\end{array}$ & Count & $\begin{array}{l}\text { Cases, } \\
\%^{\mathrm{a}}\end{array}$ & Rate $^{\text {b }}$ & $\begin{array}{l}95 \% \\
\text { CI }\end{array}$ & Count & $\begin{array}{l}\text { Cases, } \\
\%^{\mathrm{a}}\end{array}$ & Rate $^{b}$ & $\begin{array}{l}95 \% \\
\mathrm{CI}\end{array}$ & Count & $\begin{array}{l}\text { Cases, } \\
\%^{\mathrm{a}}\end{array}$ & Rate $^{\text {b }}$ & $\begin{array}{l}95 \% \\
\text { CI }\end{array}$ \\
\hline \multicolumn{17}{|c|}{ American Indian/Alaska Native } \\
\hline Northern Plains & 10 & 14.5 & 3.0 & $1.4-5.6$ & 34 & 49.3 & 9.0 & $6.2-12.6$ & 15 & 21.7 & 10.1 & $5.6-16.6$ & 10 & 14.5 & $14.2^{\mathrm{c}}$ & $6.6-26.9$ \\
\hline Alaska & d & 9.5 & 1.5 & $0.2-5.3$ & 13 & 61.9 & 7.3 & $3.9-12.6$ & $\mathrm{~d}$ & 14.3 & 4.1 & $0.8-12.1$ & d & 14.3 & 9.7 & $2.0-27.9$ \\
\hline Southern Plains & d & 3.4 & 0.9 & $0.3-2.3$ & 75 & 64.1 & $14.7^{\mathrm{C}}$ & $11.6-18.5$ & 25 & 21.4 & 10.4 & $6.7-15.4$ & 13 & 11.1 & 9.1 & $4.8-15.6$ \\
\hline Pacific Coast & 7 & 10.8 & 1.2 & $0.5-2.6$ & 35 & 53.8 & 5.3 & $3.7-7.4$ & 14 & 21.5 & 4.9 & $2.7-8.3$ & 9 & 13.8 & 7.7 & $3.5-14.9$ \\
\hline East & d & 13.3 & 1.8 & $0.2-6.3$ & 6 & 40.0 & 3.9 & $1.4-8.4$ & d & 26.7 & 6.2 & $1.7-15.8$ & d & 20.0 & 10.2 & $2.1-29.7$ \\
\hline Southwest & 7 & 7.4 & 0.9 & $0.4-1.9$ & 45 & 47.9 & 5.4 & $3.9-7.3$ & 23 & 24.5 & 6.9 & 4.4-10.4 & 19 & 20.2 & $10.7^{\mathrm{c}}$ & $6.4-16.8$ \\
\hline Total & 32 & 8.4 & 1.4 & $0.9-1.9$ & 208 & 54.6 & $7.6^{\mathrm{c}}$ & $6.6-8.8$ & 84 & 22.0 & 7.4 & $5.9-9.2$ & 57 & 15.0 & $10.0^{\mathrm{c}}$ & $7.6-13.0$ \\
\hline \multicolumn{17}{|l|}{ Non-Hispanic white } \\
\hline Northern Plains & 154 & 8.7 & 1.8 & $1.5-2.1$ & 850 & 47.9 & 6.3 & $5.9-6.7$ & 435 & 24.5 & 5.7 & $5.1-6.2$ & 336 & 18.9 & 5.1 & $4.6-5.7$ \\
\hline Alaska & d & 3.8 & 0.6 & $0.1-1.7$ & 46 & 59.0 & 4.9 & $3.6-6.6$ & 21 & 26.9 & 4.9 & $3.0-7.5$ & 8 & 10.3 & 5.2 & $2.2-10.4$ \\
\hline Southern Plains & 58 & 7.1 & 1.7 & $1.3-2.3$ & 386 & 47.1 & 8.1 & 7.4-9.0 & 191 & 23.3 & 6.4 & $5.6-7.4$ & 184 & 22.5 & 7.0 & $6.1-8.1$ \\
\hline Pacific Coast & 213 & 6.9 & 1.4 & $1.2-1.6$ & 1583 & 51.2 & 6.4 & $6.0-6.7$ & 738 & 23.9 & 5.1 & $4.7-5.4$ & 556 & 18.0 & 4.7 & $4.3-5.1$ \\
\hline East & 106 & 4.8 & 1.2 & $1.0-1.4$ & 961 & 43.5 & 6.0 & $5.6-6.3$ & 577 & 26.1 & 6.1 & $5.6-6.6$ & 566 & 25.6 & 6.4 & $5.8-6.9$ \\
\hline Southwest & 78 & 5.6 & 1.2 & $0.9-1.5$ & 627 & 44.8 & 6.1 & $5.7-6.6$ & 401 & 28.6 & 6.2 & $5.6-6.9$ & 294 & 21.0 & 5.2 & $4.6-5.8$ \\
\hline Total & 612 & 6.5 & 1.4 & $1.3-1.5$ & 4453 & 47.5 & 6.3 & $6.1-6.5$ & 2363 & 25.2 & 5.7 & $5.5-5.9$ & 1944 & 20.7 & 5.4 & $5.2-5.7$ \\
\hline
\end{tabular}

Source: Cancer registries in the Centers for Disease Control and Prevention's National Program of Cancer Registries (NPCR) and/or the National Cancer Institute's Surveillance, Epidemiology and End Results (SEER) program.

CHSDA indicates Contract Health Service Delivery Areas; IHS, Indian Health Service; 95\% CI, 95\% confidence interval.

a Percentages may not add up to $100.0 \%$ due to rounding.

b Rates are per 100,000 persons and are age-adjusted to the 2000 US standard population (19 age groups).

c The American Indian/Alaska Native rate is statistically significantly higher than the Non-Hispanic white rate $(P<.05)$.

${ }^{\mathrm{d}}$ Counts $<6$ are suppressed; if no cases were reported, then row percentages and rates could not be calculated.

Years of data and registries used: 1999 to 2004 (41 states and the District of Columbia; ${ }^{*}$ indicates states with at least 1 county designated as CHSDA): Alaska, ${ }^{*}$ Alabama, ${ }^{*}$ Arkansas, Arizona, ${ }^{*}$ California, ${ }^{*}$ Colorado, ${ }^{*}$ Connecticut, ${ }^{*}$ District of Columbia, Delaware, Florida, ${ }^{*}$ Georgia, Hawaii, Iowa, ${ }^{*}$ Idaho, ${ }^{*}$ Illinois, Indiana, ${ }^{*}$ Kentucky, ${ }^{*}$ Louisiana, ${ }^{*}$ Massachusetts, ${ }^{*}$ Maine, ${ }^{*}$ Michigan, ${ }^{*}$ Minnesota, ${ }^{*}$ Missouri, Montana, ${ }^{*}$ North Carolina, ${ }^{*}$ Nebraska, ${ }^{*}$ New Hampshire, New Jersey, New Mexico, ${ }^{*}$ Nevada, ${ }^{*}$ New York, ${ }^{*}$ Ohio, Oklahoma, ${ }^{*}$ Oregon, ${ }^{*}$ Pennsylvania, ${ }^{*}$ Rhode Island, ${ }^{*}$ Texas, ${ }^{*}$ Utah, ${ }^{*}$ Washington, ${ }^{*}$ Wisconsin, ${ }^{*}$ West Virginia, and Wyoming*; 1999 and 2002 to 2004: North Dakota*; 2001 to 2004: South Dakota*; 2003 to 2004: Mississippi* and Virginia; 2004: Tennessee.

intervals for age-adjusted rates and standardized RRs were calculated based on methods described by Tiwari et $\mathrm{al}^{34}$ using SEER*Stat 6.3.6 statistical software. $^{35}$

\section{RESULTS}

Our most important findings are summarized in Tables 1 through 3 . Table 1 demonstrates regional variations in invasive cervical cancer incidence rates, per 100,000 population, for AI/AN women that are more striking than the variation among NHW women. For the period 1999 through 2004 for AI/AN women residing in CHSDA counties, invasive cervical cancer incidence rates ranged from 14.1 in the Southern Plains to 6.9 in the Pacific Coast region. The rate for AI/AN women in the Northern Plains was also elevated (12.5) in comparison with other regions. For all regions combined, the incidence rate was 9.4 , which was significantly higher than the rate for NHW women (7.4; RR of 1.28). Rates for AI/AN females in All Counties were substantially lower than those in CHSDA counties and will not be presented for subsequent tables.

The age-specific rates for invasive cervical cancer changed most notably for AI/AN women from their 30 s and 40s, remained elevated through 64 years of age, and were highest for women aged $\geq 65$ years (Table 2). This trend was consistent for most geographic areas nationwide. The mean age at diagnosis for AI/AN women was 47.3 years versus 50.1 years for NHW women (data not shown). The differences in incidence rates for invasive cervical cancer between AI/AN women and NHW women in CHSDA counties increased with age. For AI/AN women aged $\geq 65$ years, rates were $85 \%$ higher than for NHW women. Rates for AI/AN women were highest in the oldest age group, in contrast to NHW women, who demonstrated the highest rates among women ages 30 to 49 years. For those women ages 30 years to 
49 years, Southern Plains AI/AN women had the highest rates for all the regions, but for AI/AN women in the oldest age group, Northern Plains women had the highest rates.

Stage of disease at diagnosis is shown in Table 3 for AI/AN and NHW women in CHSDA counties. Herein, we noted a lower rate and proportion of lesions diagnosed at the local stage (confined to the cervix) among AI/AN women compared with NHW women, and higher rates for regional and distant stage cervical cancer among AI/AN women compared with NHW women, for all regions combined. Although specific regions of the country demonstrated some variability with respect to stage at diagnosis, the majority of the findings were consistent with the All-Regions pattern and generally demonstrated lower rates and proportions of early invasive cancer for AI/AN women.

\section{DISCUSSION}

Our results demonstrated regional differences in incidence rates of invasive cervical cancer in AI/AN women, with women in the Northern and Southern Plains having the highest rates of the disease. Furthermore, the rates comparing AI/AN with NHW women were approximately $70 \%$ and $55 \%$ greater in the Northern and Southern Plains regions, respectively. Although rates of cervical cancer among AI/ AN women vary by region and remain overall higher than in NHW women, in New Mexico and Alaska (the only regions of the US with substantial AI/AN populations in which analyses examining long-term incidence trends have been possible), rates have decreased substantially since the 1970s. ${ }^{5,13,14}$

Studies in non-AI/AN populations have demonstrated that $>50 \%$ of incident cervical cancer cases can be attributed to no screening and another $10 \%$ to no recent screening. ${ }^{36,37}$ Rates of invasive cervical cancer in AI/AN women are currently lower than earlier published reports; the decrease in rates can be attributed, in part, to aggressive screening programs that have been put into place in numerous clinics that serve AI/AN women nationwide. The IHSinitiated cervical cancer screening in the 1960s and 1970s resulted in declining cervical cancer incidence and mortality rates in the 1980s and 1990s. ${ }^{5,13,14}$ With the establishment of the CDC's National Breast and Cervical Cancer Early Detection Program (NBCCEDP), additional resources became available to increase screening in $\mathrm{AI} / \mathrm{AN}$ women. ${ }^{38}$ Programs in all 50 states and 14 tribes (Arctic Slope Native Association Limited, Barrow, Alaska; Cherokee Nation, Tahlequah, Oklahoma; Cheyenne River Sioux
Tribe, Eagle Butte, South Dakota; Hopi Tribe, Kykotsmovi, Arizona; Kaw Nation of Oklahoma, Newkirk, Oklahoma; Mississippi Band of Choctaw Indians, Choctaw, Mississippi; Native American Rehabilitation Association of the Northwest, Portland, Oregon; Navajo Nation, Window Rock, Arizona; Poarch Band of Creek Indians, Atmore, Alabama; South East Alaska Regional Health Consortium, Sitka, Alaska; South Puget Intertribal Planning Agency, Shelton, Washington; Southcentral Foundation, Anchorage, Alaska; and Yukon-Kuskokwim Health Corporation, Bethel, Alaska) currently receive support from this program to build infrastructure and provide screening services. The prevalence of Papanicolaou (Pap) screening in all counties and all regions combined for the period 1999 through 2006 based on Behavioral Risk Factor Surveillance data as reported elsewhere in this supplement $^{39}$ remains slightly lower for AI/AN women than for NHW women $(78 \%$ vs $84 \%$, respectively). Regional prevalence estimates of Pap utilization only loosely reflect regional incidence rates; however, the observation that the Southern Plains region has the highest incidence rate of cervical cancer and the lowest attendance for Pap screening ${ }^{39}$ indicates that screening prevalence influences regional patterns of cervical cancer incidence in AI/AN women.

During a site visit to the Navajo Reservation in 1989, the American College of Obstetricians and Gynecologists' Committee on American Indian Affairs identified access to colposcopy, a part of the standard diagnostic follow-up for abnormal Pap test results, as an important unmet need. In response, the CDC began supporting the IHS to conduct colposcopy training, annual training for primary care providers to increase the availability of this service and to reduce the time interval from abnormal screening results to definitive diagnosis. ${ }^{40,41}$ Since then, the IHS has increased the availability of colposcopy nationwide by training IHS and tribal providers. The IHS program provides basic colposcopy training with a didactic course followed by a hands-on preceptorship. In addition, annual review workshops and biennial colposcopy update courses enhance the skills of new and experienced colposcopists caring for AI/AN women. As of 2007, this training has reached 284 physicians and advanced practice clinicians (certified nurse midwives, nurse practitioners, and physician assistants) from 105 IHS, tribal, and urban Indian hospitals and clinics nationwide (unpublished data). Continued diligence in education, training, and prevention services is essential to further reduce the death rate from cervical cancer. Clinicians and public health practitioners, including those involved in the 


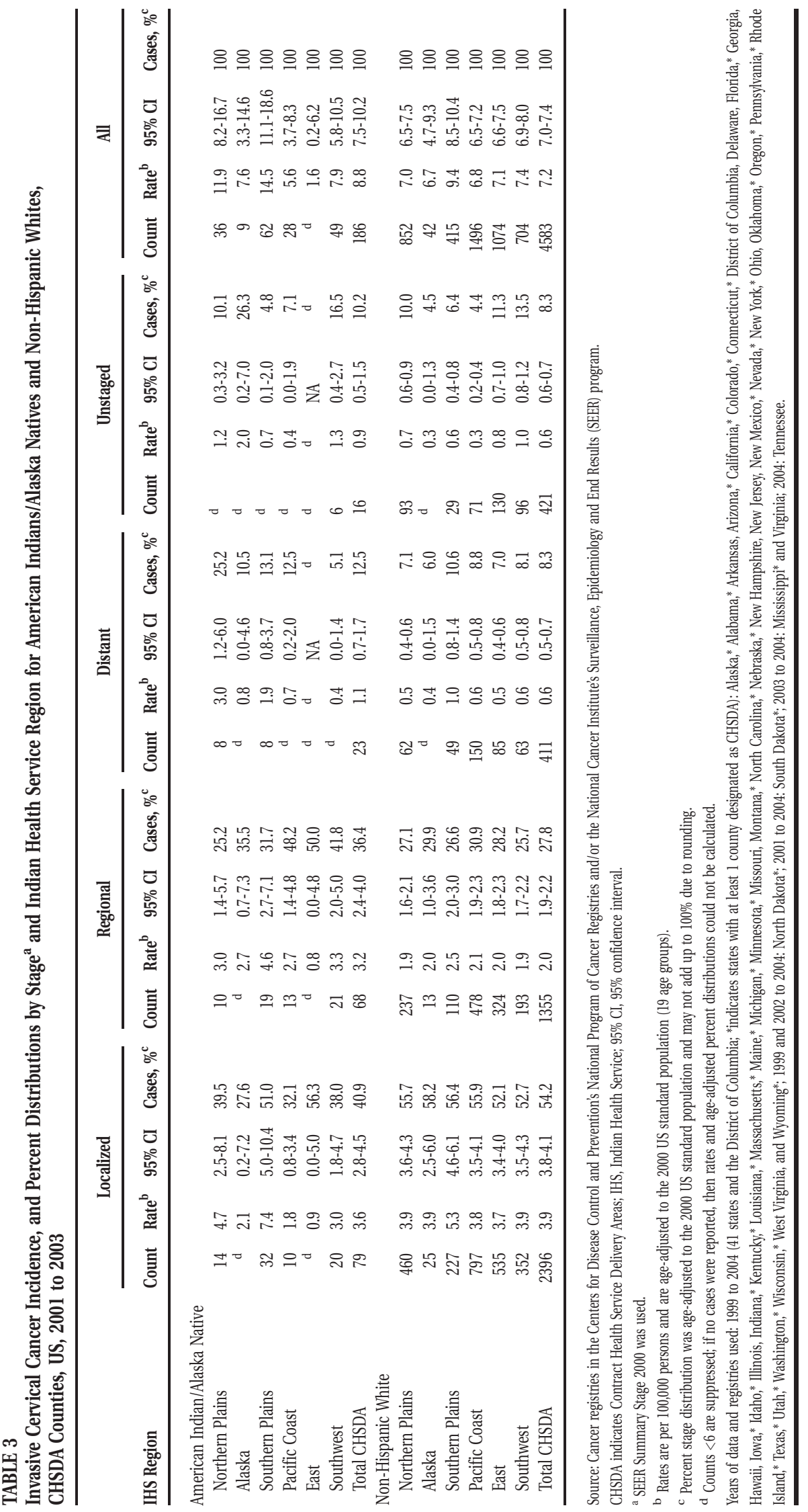


NBCCEDP, deserve much praise for their roles in lowering rates of cervical cancer, and the comparatively low rates among AI/AN women now should be viewed as a public health achievement of substantial magnitude.

Despite the progress in reducing overall cervical cancer rates, the higher overall rates in $\mathrm{AI} / \mathrm{AN}$ women and the unfavorable profile of local versus advanced stage of disease at diagnosis indicate a continued need for aggressive and comprehensive screening and timely follow-up of abnormal results. In addition to continued education and vigilance related to screening, AI/AN women should be made aware of the signs of cervical cancer (bleeding, especially after intercourse) and the critical importance of seeking immediate care if that clinical sign is apparent. Furthermore, it is critical to support screening programs with capacity to provide timely follow-up care for women with abnormal Pap smears.

Previous research in other populations has documented an increased risk of cervical cancer or of preinvasive cervical lesions among women of lower socioeconomic status. ${ }^{42,43}$ In 1 study among women in the Southwest, AI/AN women with less than a high school education and low annual income were found to be at an increased risk of developing cervical intraepithelial neoplasia (CIN). ${ }^{16}$ In a similarly designed study of risk factors for CIN among New Mexico Hispanics and NHWs, Becker et $\mathrm{al}^{44}$ also found strong risks associated with low economic status and education levels. Brinton and Fraumeni reported an inverse relation between socioeconomic indicators of income and education and risk of invasive cervical neoplasia for both black and white women. ${ }^{45}$ For the period 2000 through 2006, poverty among the AI/AN population nationally was 3 times that of the NHW population, with the highest regional prevalence of poverty noted in AI/ANs in the Southwest. ${ }^{4}$ Furthermore, AI/AN adults were less likely to have graduated from high school and were more likely to have less than a ninth grade education than NHW adults, with the Southwest and East AI/ AN populations experiencing the highest prevalence of not finishing high school. ${ }^{4}$ Economic conditions in the Plains states are also very unfavorable for AI/AN people, compared with the NHW population.

Nonetheless, from an etiologic standpoint, poverty and low levels of education are not the direct cause of abnormal cervical epithelium, but only a correlate of other factors, the most of important of which is infection with oncogenic subtypes of human papillomavirus (HPV). Finally, cigarette smoking increases the risk for cervical neoplasia. ${ }^{46}$ Because
AI/AN women have a much higher prevalence of cigarette smoking, especially in the Plains region and Alaska, ${ }^{39}$ addressing this important risk factor is critical to control a host of adverse health outcomes, including cervical cancer.

The data from the current study generally demonstrated higher age-specific cervical cancer rates for AI/AN women compared with NHW women, for most regions of the country and for most age categories that we examined. However, racial differences in rates were much less pronounced for the youngest age group of women. In some regions, for certain age groups, rates for NHW women exceeded rates for AI/AN women (see Table 3), although this observation is the exception and may reflect the instability of the AI/AN rates because of relatively small numbers of cases. The current study data do not allow us to identify those factors that explain the higher rates for NHW women than for AI/AN women in certain regions.

Our analysis indicated that AI/AN women are more commonly diagnosed with cervical cancer at a later stage than NHW women. Many factors influence the stage at which cervical cancer is diagnosed, and AI/AN women face several barriers to the early detection of cervical dysplasia and invasive cancer. Provider time pressures, health systems designed primarily for acute and episodic care, and an underfunded health system are potential barriers IHS and tribal providers may face. In addition, cultural reluctance to access Western medicine for nonacute health problems and transportation difficulties are factors commonly cited as barriers to cancer screening by $\mathrm{AI} / \mathrm{AN}$ individuals. ${ }^{47-50}$

Although $2 \mathrm{HPV}$ vaccines designed to prevent cervical neoplasia are being tested, only 1 , the quadrivalent vaccine, is now licensed in the US and recommended for use among females ages 9 years to 26 years. In some parts of the country, health administrators are aggressively promoting vaccine uptake among AI/AN patients (unpublished data). The Vaccines for Children Program (VFC) is a federal entitlement program allowing private and public providers who participate in the VFC program to vaccinate eligible children with federally purchased vaccines as approved by the CDC's Advisory Committee on Immunization Practices. Children aged $\leq 19$ years who are Medicaid eligible, uninsured, AI/AN (as defined by the Indian Health Services Act), are eligible to receive vaccine from providers through the VFC program. ${ }^{51}$ We will not have adequate information to determine whether the vaccine will reduce cervical cancer rates for many years, in AI/AN (or other) populations, but the vaccine could have a more 
immediate impact on the incidence of abnormal cytology, the number of follow-up procedures, and cervical dysplasia.

As HPV vaccine development expands to include other HPV types that cause cervical neoplasia, greater reductions in preinvasive lesions can be expected. Monitoring the HPV types infecting AI/AN women will help determine whether vaccination or newer technologies in screening are having an impact. In collaboration with the New Mexico Department of Health, investigators in New Mexico have developed a statewide Pap smear registry. Linkage of this registry to the New Mexico statewide immunization information system and, eventually, to the New Mexico Tumor Registry will be important to determining the efficacy, effectiveness, and safety of the HPV vaccine. The support and partnership of tribes and participation of AI/AN women will be crucial to evaluate the cervical cancer control efforts in that state (unpublished data). Researchers in Alaska plan an array of activities focusing on implementing and monitoring the HPV vaccine in AI/AN women. These include educating the public regarding the vaccine, monitoring vaccination coverage, measuring the impact on rates of dysplasia and cancer, evaluating antibody duration, studying the HPV types found in women undergoing colposcopy, and determining the economic impact of the vaccine (unpublished data).

There are several important limitations to our analyses to consider when interpreting these results. These include incidence rates based on small case numbers, particularly when data are presented by region and age group. Although data on screening prevalence for AI/AN women reported by Steele et al are presented elsewhere in this supplement, ${ }^{39}$ we do not know the patterns of adequate follow-up or the distribution of oncogenic HPV subtypes that may determine differences in cervical cancer between AI/ AN and NHW women. Furthermore, we do not know the proportion of AI/AN women or NHW women who have undergone hysterectomies and are no longer at risk for cervical disease.

Previous studies have shown that many AI/AN were misclassified as another race in cancer registry data and that the extent of misclassification varied by registry. ${ }^{18-21}$ Although linkages between cancer registry data and the IHS patient registration database improve the race classification for AI/AN cases, AI/AN persons who are not members of the federally recognized tribes, live primarily in urban settings, live long distances from IHS facilities, live in counties other than those designated as CHSDA, or are not eligible for IHS services are under-represented in the
IHS database. Additional details regarding the misclassification of $\mathrm{AI} / \mathrm{AN}$ race are available elsewhere in this supplement. ${ }^{16}$

Because we restricted the majority of the analyses to CHSDA counties, in which only $56 \%$ of AI/AN live and which tend to be located in more rural areas and Western states (Fig. 1), the results should not be generalized to all AI/AN (or NHW) women in the US. In addition, the percentage of the AI/AN population residing in CHSDA counties varies greatly among the 6 IHS regions. The percentage is particularly low in the East region (15\%).

In summary, the results of the current study indicate a much improved picture of reduced incidence of invasive cervical cancer in AI/AN women. Nonetheless, the rates in the Northern and Southern Plains regions noted during the study period are higher than the rates for AI/AN women in other regions or for NHW women; furthermore, AI/AN women in general had proportionally more late-stage diagnoses. For these reasons, additional steps must be taken to decrease further the rates in AI/AN women. Because smoking is an independent risk factor for cervical cancer, tobacco use prevention efforts among $\mathrm{AI} / \mathrm{AN}$ populations is essential, in addition to increasing the uptake of screening among AI/AN women. Although the HPV vaccine may have an effect in rate reduction in future generations, aggressive screening programs must remain in place. Vigilance concerning screening on the part of clinicians and their AI/AN patients clearly remains an important approach to cervical cancer control. For more information about cervical cancer, please visit www.cdc.gov/cancer/gynecologic.

\section{REFERENCES}

1. Norsted TL, White E. Cancer incidence among native Americans of western Washington. Int J Epidemiol. 1989; 18:22-27.

2. Lanier AP, Bulkow LR, Ireland B. Cancer in Alaskan Indians, Eskimos, and Aleuts, 1969-83: implications for etiology and control. Public Health Rep. 1989;104:658-664.

3. Espey DK, Paisano RE, Cobb N. Cancer mortality among American Indians and Alaska Natives: regional differences, 1994-1998. Rockville, MD: Indian Health Services; 2003.

4. Espey $\mathrm{DK}, \mathrm{Wu} \mathrm{XC}$, Swan J, et al. Annual report to the nation on the status of cancer, 1975-2004, featuring cancer in American Indians and Alaska Natives. Cancer. 2007; 110:2119-2152.

5. Becker TM, Wheeler CM, Key CR, Samet JM. Cervical cancer incidence and mortality in New Mexico's Hispanics, American Indians, and non-Hispanic whites. West $\mathrm{J}$ Med. 1992;156:376-379.

6. Jordan SW, Key CR. Carcinoma of the cervix in Southwestern American Indians: results of a cytologic detection program. Cancer. 1981;47:2523-2532. 
7. Lanier AP, Kelly JJ, Smith B, et al. Alaska Native cancer update: incidence rates 1989-1993. Cancer Epidemiol Biomarkers Prev. 1996;5:749-751.

8. Leman RF, Espey D, Cobb N. Invasive cervical cancer among American Indian women in the Northern Plains, 1994-1998: incidence, mortality, and missed opportunities. Public Health Rep. 2005;120:283-287.

9. Benard VB, Lee NC, Piper M, Richardson L. Race-specific results of Papanicolaou testing and the rate of cervical neoplasia in the National Breast and Cervical Cancer Early Detection Program, 1991-1998 (United States). Cancer Causes Control. 2001;12:61-68.

10. Cobb N, Paisano RE. Patterns of cancer mortality among Native Americans. Cancer. 1998;83:2377-2383.

11. Espey D, Paisano R, Cobb N. Regional patterns and trends in cancer mortality among American Indians and Alaska Natives, 1990-2001. Cancer. 2005;103:1045-1053.

12. Jordan SW, Sopher RL, Key CR, Brylinski D, Huang J. Carcinoma of the cervix in Southwestern American Indian women. Cancer. 1972;29:1235-1241.

13. Chao A, Becker TM, Jordan SW, Darling R, Gilliland FD, Key CR. Decreasing rates of cervical cancer among American Indians and Hispanics in New Mexico (United States). Cancer Causes Control. 1996;7:205-213.

14. Lanier AP, Kelly JJ, Maxwell J, McEvoy T, Homan C. Cancer in Alaska Native people, 1969-2003. Alaska Med. 2006;48:30-59.

15. Becker TM, Wheeler CM, McPherson RS, et al. Risk factors for cervical dysplasia in Southwestern American Indian women: a pilot study. Alaska Med. 1993;35:255-263.

16. Schiff M, Becker TM, Masuk M, et al. Risk factors for cervical intraepithelial neoplasia in Southwestern American Indian women. Am J Epidemiol. 2000;152:716-726.

17. Davidson M, Schnitzer PG, Bulkow LR, et al. The prevalence of cervical infection with human papillomaviruses and cervical dysplasia in Alaska Native women. J Infect Dis. 1994;169:792-800.

18. Frost F, Taylor V, Fries E. Racial misclassification of Native Americans in a surveillance, epidemiology, and end results cancer registry. J Natl Cancer Inst. 1992;84:957-962.

19. Kwong S, Perkings C, Snipes K, Wright W. Improving American Indian cancer data in the California Cancer Registry by linkage with the Indian Health Service. J Registry Manage. 1998;25:17-20.

20. Becker TM, Bettles J, Lapidus J, et al. Improving cancer incidence estimates for American Indians and Alaska Natives in the Pacific Northwest. Am J Public Health. 2002; 92:1469-1471.

21. Harwell TS, Miller SH, Lemons DL, Helgerson SD, Gohdes D. Cancer incidence in Montana: rates for American Indians exceed those for whites. Am J Prev Med. 2006;30: 493-497.

22. Espey DK, C W, MA J, Miller BA, Johnson C, Becker TM. Methods for improving cancer surveillance data in American Indian and Alaska Native Populations. Cancer. 2008; 113(suppl).

23. US Cancer Statistics Working Group. United States cancer statistics: 2004 incidence. Atlanta, GA: Department of Health and Human Services, Centers for Disease Control and Prevention and National Cancer Institute; 2007.

24. US Cancer Statistics Working Group. United States cancer statistics: 2003 incidence and mortality. Atlanta, GA: U.S. Department of Health and Human Services, Centers for Disease Control and Prevention and National Cancer Institute; 2006
25. Fritz A, Percy C, Jack A. International classification of diseases of oncology. Geneva, Switzerland: World Health Organization; 2000.

26. Office of Management and Budget. Revisions to the Standards for the Classification of Federal Data on Race and Ethnicity. Federal Register Notice: October 30, 1997. Available at: http://www.whitehouse.gov/omb/fedreg/1997 standards.html Accessed on July 8, 2008.

27. Link Plus. A suite of publicly available software programs for collecting and processing cancer registry data. Atlanta, GA: Centers for Disease Control and Prevention; 2005. Available at: http://www.cdc.gov/cancer/npcr/tools/ registryplus/lp.htm Accessed on July 8, 2008.

28. Jim MA, Espey DK, Wiggins C, Cobb N, Wingo PA. Racial Misclassification of American Indians Residing Near IHS Facilities. Poster P-47, Final Program and Abstracts. Presented at the North American Association of Central Cancer Registries Conference, Regina, Saskatchewan, Canada, June 13-16, 2006.

29. Denny CH, Holtzman D, Cobb N. Surveillance for health behaviors of American Indians and Alaska Natives. Findings from the Behavioral Risk Factor Surveillance System, 1997-2000. MMWR Surveill Summ. 2003;52:1-13.

30. Howe HL, Jamison M, Havener L, Chen VW, Ries LAG. Sitespecific comparison of summary stage 1977 and summary stage 2000 coding. Available at: http://www.naaccr.org/ index.asp?COL_SectionKey=11\&COL_ContentID=397 Accessed on July 8, 2008.

31. Phillips J, ed. Summary stage: data effects of the changes in 2000. Available at: http://www.naaccr.org/filesystem/ pdf/Summary\%20Stage\%20Report\%201-21-04b.pdf.

32. Ingram DD, Parker JD, Schenker N, et al. United States Census 2000 population with bridged race categories. Vital Health Stat 2. September 2003;2.

33. National Cancer Institute. Surveillance, Epidemiology, and End Results (SEER) Program. Statistical Resources. U.S. population data 1969-2004. Available at: http://seer.cancer. gov/resources. Accessed on July 8, 2008.

34. Tiwari RC, Clegg LX, Zou Z. Efficient interval estimation for age-adjusted cancer rates. Stat Methods Med Res. 2006;15: 547-569.

35. Surveillance Research Program. SEER*Stat software. Bethesda, MD: National Cancer Institute, DCCPS, Surveillance Research Program, Cancer Statistics Branch; 2007. Available at: http://www.seer.cancer.gov/seerstat. Accessed on July 8, 2008.

36. Sung H, Kearney K, Miller M, Kinney W, Sawaya G, Hiatt R. Papanicolaou smear history and diagnosis of invasive cervical carcinoma among members of a large prepaid health plan. Cancer. 2000;88:2283-2289.

37. Sawaya GF, Grimes DA. New technologies in cervical cytology screening: a word of caution. Obstet Gynecol. 1999;94: 307-310.

38. Henson RM, Wyatt SW, Lee NC. The National Breast and Cervical Cancer Early Detection Program: a comprehensive public health response to 2 major health issues for women. J Public Health Manag Pract. 1996;2:36-47.

39. Steele C, Cardinez C, Richardson L, Tom-Orme L, Shaw K. Surveillance for health behaviors of American Indians and Alaska Natives: findings from the Behavioral Risk Factor Surveillance System, 2000-2006. Cancer. 2008;113(5 suppl):1131-1141.

40. Waxman AG. Colposcopy training for IHS providers: a strategy for improving cervical cancer screening in Native Americans. IHS Provider. 1992;17:3. 
41. Waxman AG, Inkret W. Teaching colposcopy on the Navajo reservation. Colposcopist. 1991;23:2.

42. Brown J, Harding S, Bethune A, Rosato M. Incidence of health of the nation cancers by social class. Popul Trends. Winter 1997:40-47,49-77.

43. Faggiono F, Partanen T, Kogevinas M. Socioeconomic differences in cancer incidence and mortality IARC Scientific Publ. No. 138. Lyon, France: International Agency for Research on Cancer;1997:65-176.

44. Becker TM, Wheeler CM, McGough NS, et al. Sexually transmitted diseases and other risk factors for cervical dysplasia among Southwestern Hispanic and non-Hispanic white women. JAMA. 1994;271:1181-1188.

45. Brinton LA, Fraumeni JF Jr. Epidemiology of uterine cervical cancer. J Chronic Dis. 1986;39:1051-1065.

46. Appleby P, Beral V, Berrington de Gonzalez A, et al. Carcinoma of the cervix and tobacco smoking: collaborative reanalysis of individual data on 13,541 women with carcinoma of the cervix and 23,017 women without carcinoma of the cervix from 23 epidemiological studies. Int J Cancer. 2006;118:1481-1495.

47. Coe K, Martin L, Nuvayestewa L, et al. Predictors of Pap test use among women living on the Hopi reservation. Health Care Women Int. 2007;28:764-781.

48. Michalek AM, Mahoney MC, Papas M, Tenney M, Burhansstipanov L. Tribal-based cancer control activities among Alaska Natives: services and perceptions. Alaska Med. 1996;38:59-64,83.

49. Canales M. Taking care of self: health care decision making of American Indian women. Health Care Women Int. 2004; 25:411-435.

50. Novins DK, Beals J, Moore LA, Spicer P, Manson SM. Use of biomedical services and traditional healing options among American Indians: sociodemographic correlates, spirituality, and ethnic identity. Med Care. 2004;42:670679.

51. Santoli JM, Rodewald LE, Maes EF, Battaglia MP, Coronado VG. Vaccines for Children program, United States, 1997. Pediatrics. 1999;104:e15. 